\title{
Functionally sustainable wireless sensor network technologies aspects analysis
}

\author{
*A. V. Sobchuk ${ }^{1}$, V. V. Sobchuk ${ }^{2}$, O. V. Barabash ${ }^{3}$, I. Liashenko ${ }^{3}$ \\ ${ }^{1}$ Taras Shevchenko National University of Kyiv, Kyiv, Ukraine \\ ${ }^{2}$ Lesya Ukrainka Eastern European National University, Lutsk, Ukraine \\ ${ }^{3}$ State University of Telecommunications, Kyiv, Ukraine \\ ${ }^{4}$ National Economic University named after Vadym Hetman, Kyiv, Ukraine \\ *Corresponding author. E-mail: anri.sobchuk@gmail.com
}

Paper received 25.01.19; Accepted for publication 02.02.19.

https://doi.org/10.31174/SEND-NT2019-193VII23-11

\begin{abstract}
In the article wireless technologies of information transmission are considered and their comparative analysis is given, the main advantages and disadvantages are considered. The work is devoted to the systematization of wireless communication technologies in the context of the functional stability of the networks on which they are based. Since such networks can be created using various wireless technologies, they need to have the ability to self-organize, to ensure the network's stable operation and its target functions. At the same time, various network technologies provide various characteristics of their functioning (use of computing resources, energy consumption, speed and range of data transmission, etc.). Therefore, the choice of technology is primarily due to the tasks that the network must solve. Ensuring the functional stability of the network, regardless of the technologies used in its construction, will reduce human intervention, operating costs and provide the necessary level of reliability of the data observed.
\end{abstract}

Keywords: wireless sensor network, functional stability, optimization.

Introduction. Wireless sensor networks penetrate more and more into all areas of industry and human activities every year. Currently, they are widely used not only for data collection and processing of industrial objects, but also in the ordinary everyday life on the Internet [1]. This is due to many industry standards developed by various international organizations such as the International Organization for Standardization (ISO), the Institute of Electrical and Electronics Engineers (IEEE), the Internet Information Network (IETF) and the International Telecommunication Union (ITU). The results of leading industry standardization be- came known standards IEEE 802.15.4, which determine the features of construction of networks with low bandwidth [2].

Today, ZigBee radio devices have everything they need for fast information distribution: digital integrated interfaces, cheap network devices with built-in routing features, and several networking technologies. Connected to a wireless network, sensors can track environmental parameters or physiological characteristics of a person. Monitoring can be carried out on a very large area, because the sensors transmit information chain - from neighbor to neighbor. The technology allows them to work for years (even decades) without replacing batteries [3].
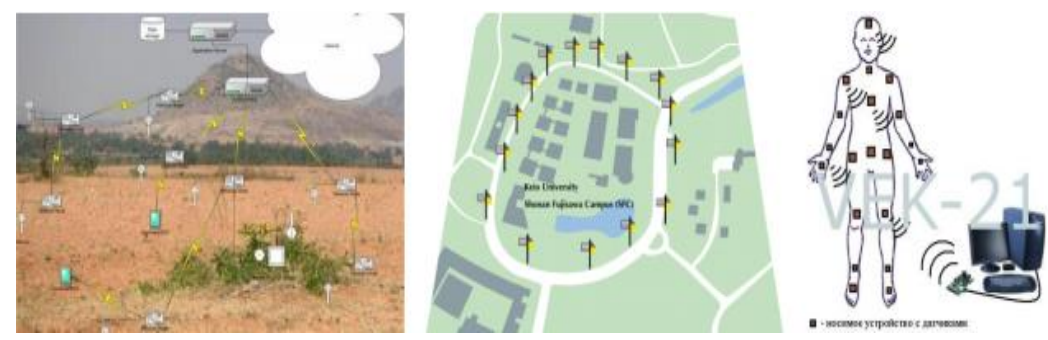

Figure 1 - Wireless sensor networks application areas.

Research results. As already noted, wireless transmitters often operate on the basis of IEEE802.15.4 and the ZigBee specification, which, consuming little electricity, can transmit information on average $30-50 \mathrm{~m}$. The most popular detectors are temperature sensors [4], humidity [5] and accelerometers [6]. Depending on the application, developers add or use additional or peripheral devices (LEDs, SPI / I2C / UART interfaces, etc.).

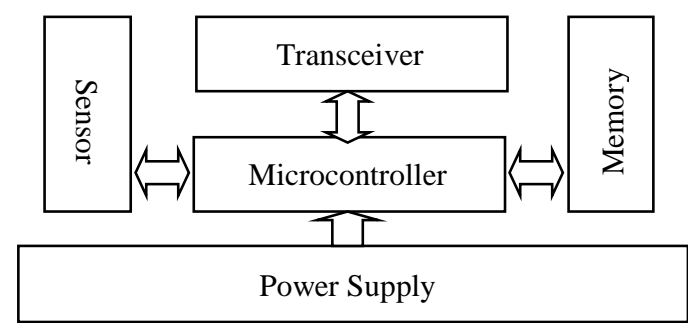

Figure 2 - The structure of the construction of the wireless sensor module
Networks that have the ability to self-organization can be created using a variety of wireless technologies. At the same time, different network technologies provide different characteristics of their operation (use of computing resources, energy consumption, speed and data transmission distance, etc.). Therefore, the choice of technology in the first place is determined by the tasks that the network must solve.

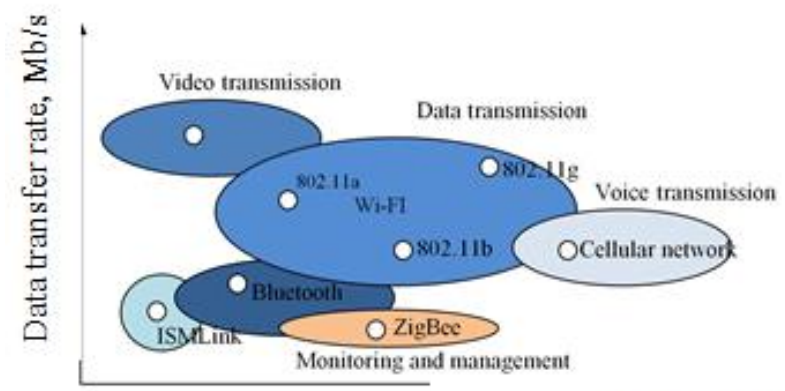

Technology

Figure 3 - Comparative analysis of wireless data standards 
The figure shows the most widely used wireless data transmission technologies. The analysis clearly demonstrates that ZigBee's wireless data technology provides the best performance of computing resources and energy efficiency. In addition, the data transfer rate provided by this technology is sufficient to transmit information from sensors as evidenced by the following data:

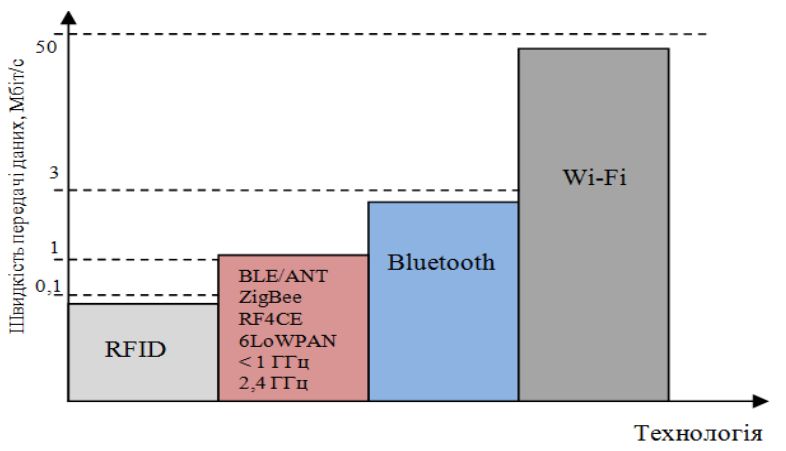

Figure 4 - Characteristics of data rates for various wireless technologies
So, the most optimal way to build wireless sensor nodes is to use the standard Zigbee. The received electrical signals from the sensor are often not ready for the calculations, so they pass through the signal transformation stage. At this stage, a series of transformations may be made, necessary for further use of the signal. For example, a signal often requires amplification, to increase amplitude, then filters are used to eliminate unwanted noise at certain frequencies. The transformed signal is transformed using an ADC into a digital signal. Thus, the signal is available in digital form and is ready for further calculation, storage and visualization.

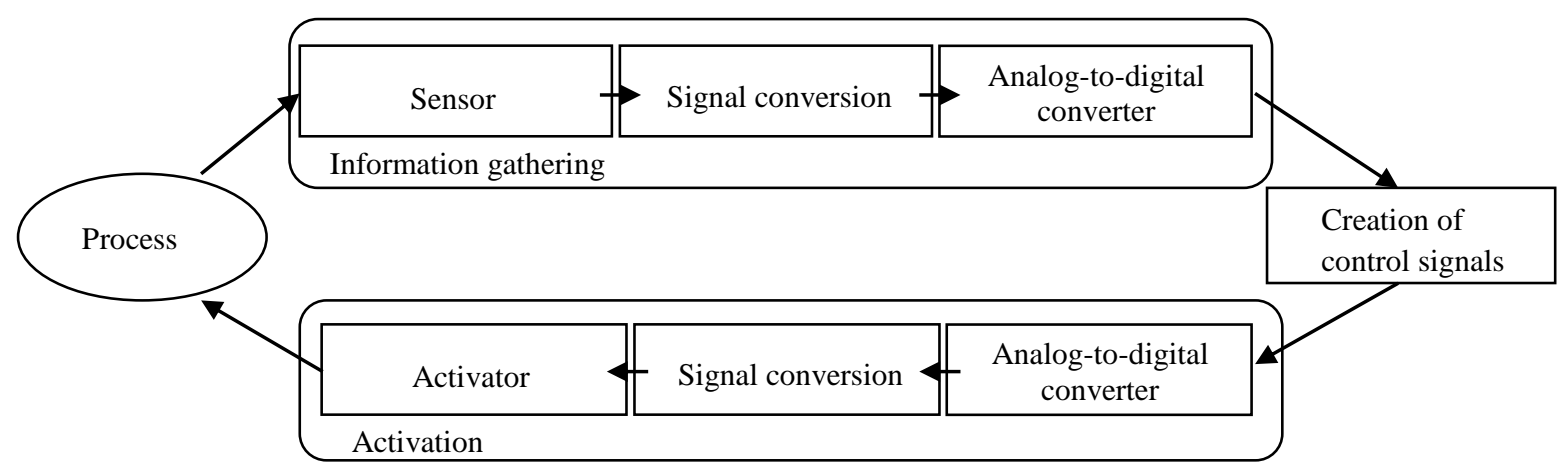

Figure 5 - The process of data collection and process activation in sensory networks

The relationship between the information transmitted and received by the sensors is described by the inverse-square law [7]:

$$
P_{p w r} \sim \frac{P_{t r}}{d^{2}}
$$

where:

$P_{p w r}$ - the power of the received signal;

$P_{t r}-$ the power of the transmitted signal;

$d$ - the distance between the receiver and the transmitter.

The distance between the nodes can be estimated based on the information about the output power of the transmitter, receiver sensitivity and antenna characteristics, taking into account empirical information.

For theoretical estimates, the Vries formula for free space is used:

$$
\begin{aligned}
& P_{r}=\frac{P_{t} \cdot G_{t} \cdot G_{r} \cdot \lambda^{2}}{(4 \pi d)^{2}} \\
& d=\frac{\lambda}{4 \pi} \cdot \sqrt{\frac{P_{t} \cdot G_{t} \cdot G_{r}}{P_{r}}}
\end{aligned}
$$$$
P_{r}=P_{t}+G_{t}+20 \cdot \log \left(\frac{\lambda}{4 \pi}\right)-20 \log d
$$

where:

$P_{t}-$ transmitter power

$P_{r}-$ signal sensitivity
$G_{t}, G_{r}$ - coefficients of amplification of antennas transmit-receive information

$d$ - the distance between the nodes

$\lambda$ - wavelength

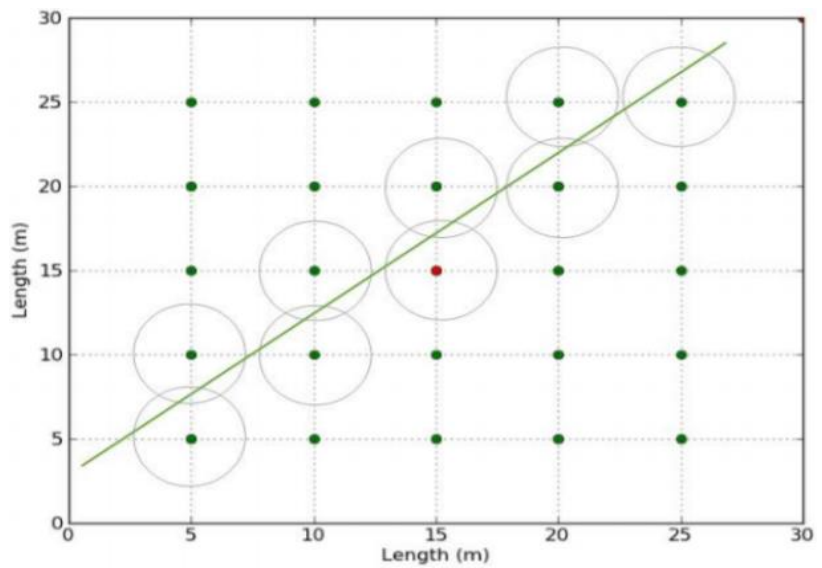

Figure 6 - Tracing a wireless sensor network of a target moving along a given linear trajectory

The whole set of sensors is integrated into a wireless network that is connected to the public communication network. It provides a wide range of control and management services for enterprises, production sites, moving objects, etc.

Conclusions. The analysis of technologies and aspects of analysis of wireless sensor networks is carried out in the 
work. Since such networks can be created using various wireless technologies, they need to have the ability to selforganize, to ensure the stable operation of the network and the fulfillment of its target functions. At the same time, different network technologies provide different characteristics of their operation (use of computing resources, energy consumption, speed and data transmission distance, etc.). There- fore, the choice of technology in the first place is determined by the tasks that the network must solve. Ensuring the functional stability of the network, regardless of the technologies used in its construction, will reduce human intervention, operating costs and provides the necessary level of reliability of the observed data.

\section{REFERENCES}

1. Recommendation Y.2060 "Overview of the Internet of things" // International Telecommunication Union Telecommunication Standardization Sector, 2012.

2. I. B. Galelyuka Modelyuvannya bezdrotovih sensory shimzh. Komp'yuterny zasobi, izmenzhi that sistemi. - 2015. - No. 14. - P 141-150. - Access mode: http://nbuv.gov.ua/UJRN/Kzms_2015_14_18.

3. R. S. Kolodiy, O. V. Timchenko. Methods of Sensing Sensitive Measures for Mobile Monitoring ECG. Journal of Lviv Polytechnic National University, №645, 2009, 46-55.

4. A. G. Finogeev, V. B. Dilman, V. A. Maslov. Operational remote monitoring in the city heating system based on wireless sensor networks. Izvestiya Vysshikh Uchebnykh Zavedenii. The Volga region. Engineering science, № 3, 2010, 27-36.

5. M. I. Gorlov. Microelectronic humidity sensor of surfacecondensation type, Patent of the Russian Federation, 2224246. Pub. 02.20.2004. Bul. №5. 107.

6. E. V. Barbasova, T. A. Vstavskaya. Construction of control systems for complex outdoor lighting complexes. Vesnik SUSU, № 23, 2011, 98-101.

7. I.M. Smurygin. The concept of the organization of wireless sensor networks and their application. Youth scientific and technical bulletin / FGBOU HPE "MSTU. N.E. Bauman ", 2012, № 9 